\title{
BMJ Open Does enhanced physical rehabilitation following intensive care unit discharge improve outcomes in patients who received mechanical ventilation? $A$ systematic review and meta-analysis
}

\author{
Shunsuke Taito, ${ }^{\circ}$ Kota Yamauchi, ${ }^{2}$ Yasushi Tsujimoto, ${ }^{3,4}$ Masahiro Banno, ${ }^{5,6}$ \\ Hiraku Tsujimoto, ${ }^{7}$ Yuki Kataoka ${ }^{8,9}$
}

To cite: Taito S, Yamauchi K, Tsujimoto Y, et al. Does enhanced physical rehabilitation following intensive care unit discharge improve outcomes in patients who received mechanical ventilation? A systematic review and meta-analysis. BMJ Open 2019;9:e026075. doi:10.1136/ bmjopen-2018-026075

- Prepublication history and additional material for this paper are available online. To view these files, please visit the journal online (http://dx.doi org/10.1136/bmjopen-2018026075).

Received 15 August 2018 Revised 22 March 2019 Accepted 17 May 2019
Check for updates

(C) Author(s) (or their employer(s)) 2019. Re-use permitted under CC BY-NC. No commercial re-use. See rights and permissions. Published by BMJ.

For numbered affiliations see end of article.

Correspondence to

Dr Shunsuke Taito;

shutaitou@hiroshima-u.ac.jp

\section{ABSTRACT}

Objective We aimed to determine whether enhanced physical rehabilitation following intensive care unit (ICU) discharge improves activities-of-daily-living function, quality of life (QOL) and mortality among patients who received mechanical ventilation in the ICU.

Design Systematic review and meta-analysis using the Grading of Recommendations Assessment, Development and Evaluation (GRADE) approach.

Data sources MEDLINE, Embase, CENTRAL, PEDro and WHO International Clinical Trials Registry Platform searched through January 2019.

Eligibility criteria for selecting studies We included randomised controlled trials assessing the effect of postICU rehabilitation designed to either commence earlier and/or be more intensive than the protocol employed in the control group. Only adults who received mechanical ventilation for $>24$ hours were included.

Data extraction and synthesis Two independent reviewers extracted data and assessed risk of bias. Standard mean differences (SMDs) with 95\% Cls were calculated for Q0L, and pooled risk ratios (RRs) with $95 \%$ Cls are provided for mortality. We assessed heterogeneity based on $\mathrm{I}^{2}$ and the certainty of evidence based on the GRADE approach.

Results Ten trials (enrolling 1110 patients) compared physical rehabilitation with usual care or no intervention after ICU discharge. Regarding QOL, the SMD $(95 \% \mathrm{Cl})$ between the intervention and control groups for the physical and mental component summary scores was 0.06 $(-0.12$ to 0.24$)$ and $-0.04(-0.20$ to 0.11$)$, respectively. Rehabilitation did not significantly decrease long-term mortality (RR 1.05, 95\% Cl 0.66 to 1.66). The analysed trials did not report activities-of-daily-living data. The certainty of the evidence for QOL and mortality was moderate.

Conclusions Enhanced physical rehabilitation following ICU discharge may make little or no difference to QOL or mortality among patients who received mechanical ventilation in the ICU. Given the wide Cls, further studies are needed to confirm the efficacy of intensive post-ICU rehabilitation in selected populations.

PROSPERO registration number CRD42017080532.
Strengths and limitations of this study

- This is the first meta-analysis focused on enhanced physical rehabilitation to review randomised controlled trials in which the study intervention was conducted only after intensive care unit discharge.

- The conclusions are based on moderate-certainty evidence.

- The main limitations of this meta-analysis are that (i) few studies had a follow-up $>6$ months and (ii) medical resources and costs associated with each intervention were not considered.

- We employed rigorous methodology that followed a protocol developed a priori according to the Preferred Reporting Items for Systematic Reviews and Meta-Analyses statement, and used the Grading of Recommendations Assessment, Development and Evaluation approach in the review process.

\section{INTRODUCTION}

In critically ill patients, rehabilitation mainly aims to enhance quality of life (QOL) by improving activities-of-daily-living (ADL) function, ${ }^{12}$ which may be severely impaired also due to postintensive care syndrome (PICS). ${ }^{3-5}$ According to the guidelines issued by the National Institute for Health and Care Excellence, provision of rehabilitation should be seamlessly integrated with the patient's transition from the intensive care unit (ICU) to the ward and then to out-of-hospital care. ${ }^{6}$ However, at the time the guidelines were issued, there was little evidence from clinical trials to support the use of enhanced physical rehabilitation following ICU discharge. Some experts do recommend physical rehabilitation following ICU discharge to improve ADL function and QOL. ${ }^{7}$ With regard to sepsis survivors, the findings of a large observational 
study suggested that physical rehabilitation following ICU discharge improves long-term mortality. ${ }^{89}$

A recent systematic review by Connolly et $a l^{10}$ focused on randomised controlled trials (RCTs) regarding the effectiveness of enhanced exercise rehabilitation following ICU discharge in adult ICU survivors who had been mechanically ventilated for longer than 24 hours in the ICU. Despite the comprehensive search, this previous systematic review included only six RCTs with conflicting results, and no clear effect of the intervention on QOL, mortality, functional exercise capacity or incidence of adverse events could be established at the time. Additionally, ADL, pain, return-to-work rate, muscle strength and duration of delirium were not considered in that review. ${ }^{10}$ Several RCTs assessing the effect of enhanced physical rehabilitation following ICU discharge on clinically relevant outcomes ${ }^{1-15}$ have been published since Connolly et al conducted their Cochrane review. ${ }^{10}$ Therefore, in the present study, we aimed to re-evaluate the available literature and determine whether enhanced physical rehabilitation following ICU discharge improves clinically relevant outcomes among critically ill adults who received mechanical ventilation.

\section{MATERIALS AND METHODS}

\section{Compliance with reporting guidelines}

Using a prespecified protocol (PROSPERO registry ID: CRD42017080532), ${ }^{16}$ we conducted a systematic review of the relevant literature in agreement with the recommendations listed in the Cochrane Handbook ${ }^{17}$ and the Preferred Reporting Items for Systematic Reviews and Meta-Analysis (PRISMA) guidelines. ${ }^{18}$ We confirmed that this systematic review was PRISMA-compliant by consulting the PRISMA 2009 checklist ${ }^{19}$ (details provided in online supplementary file 1 ).

\section{Research question and eligibility criteria}

The research question addressed in this study was: 'Does enhanced physical rehabilitation following ICU discharge result in improved QOL, ADL function and mortality (compared with those achievable with usual care) among patients who received mechanical ventilation in the ICU?' We included all published and unpublished prospective RCTs involving adult human subjects (age $\geq 18$ years) who had been discharged from an ICU or critical care environment after a stay of at least 48 hours during which mechanical ventilation was provided for at least 24 hours. Crossover trials, as well as cluster-randomised, quasi-randomised and non-randomised trials were excluded. Studies were included regardless of the intervention setting (in-hospital or out-of-hospital), follow-up duration and country of origin. We included patients of any sex and race, but excluded those receiving palliative care and those with head or spinal cord injuries, or unstable fracture diminishing mobility.

Intervention was defined as any protocolised rehabilitation following ICU discharge, designed to either commence earlier and/or be more intensive than the care received by the control group. To determine whether enhanced physical rehabilitation following ICU discharge improved clinically relevant outcomes, we excluded studies in which the patients in the intervention group received earlier and/or more intensive physical rehabilitation (compared with the care received by the control group) during their stay in the ICU. However, while we excluded studies in which enhanced rehabilitation was provided in the ICU, we did not exclude studies in which the same rehabilitation programme was provided in the ICU as standard care for both the intervention group and the control group. Protocolised rehabilitation consisting of one or more of the following activities was considered as a form of enhanced physical rehabilitation: neuromuscular stimulation, inspiratory or respiratory muscle training, passive range-of-motion exercise, cycle ergometer exercise, active-assisted exercises, active range-ofmotion exercises, bed mobility activities (eg, bridging, rolling, lying-to-sitting exercise), ADL training, transfer training, pregait exercises (including marching in place) and walking exercise.

\section{Outcomes of interest}

The primary outcomes were QOL, ADL function and mortality. Secondary outcomes included functional exercise capacity, pain, return-to-work rate, muscle strength, duration of delirium and incidence of adverse events (defined by the trialists). We defined the intervention outcomes according to the timing of their evaluation postintervention, as short-term (evaluated at 28-35 days) or long-term (evaluated at 6 months).

\section{Search strategy and selection of studies}

We searched the Cochrane Central Register of Controlled Trials (CENTRAL), MEDLINE via PubMed, Excerpta Medica Database (EMBASE) via Elsevier, the Physiotherapy Evidence Database (PEDro) and the WHO International Clinical Trials Registry Platform (WHO ICTRP) via their dedicated search portal. The search, which employed a set of suitable search terms (details provided in online supplementary file 2), was performed in December 2017 and updated in January 2019. We handsearched reference lists for the guidelines for rehabilitation after critical illness. ${ }^{6}$ We attempted to identify other relevant research by hand-searching the reference lists of the studies returned by the search and those of articles citing such studies (based on citation information from the Web of Science). If the database entry for a candidate study did not contain the necessary information, we contacted the study authors. Two reviewers (ST and $\mathrm{KY}$ ) independently screened the title and abstract of each study returned by the search to determine whether the inclusion criteria were met. The two reviewers performed a full-text review to assess the eligibility of each candidate study. Disagreement was resolved by discussion between the two reviewers, occasionally with arbitration by a third reviewer (YK). 


\section{Data abstraction and quality assessment}

Two reviewers (ST and KY) independently abstracted trial-level data using prespecified forms. Disagreements regarding data extraction were resolved through discussions. Where necessary, we contacted the authors of studies that did not provide sufficient information. The risk of bias in each study was assessed independently by two reviewers (ST and $\mathrm{KY}$ ) using the Cochrane risk-of-bias assessment tool. ${ }^{17}$ Differences in opinion regarding the assessment of risk of bias were resolved through discussion between the two reviewers, occasionally with arbitration by a third reviewer $(\mathrm{KY})$.

\section{Data analysis}

All analyses were conducted using the Cochrane Review Manager software (RevMan V.5.3; Cochrane Collaboration, Copenhagen, Denmark). For the dichotomous variables of mortality and return-to-work rate, pooled risk ratios (RRs) with $95 \%$ CIs are provided. For continuous outcomes including QOL scores, ADL function scores, pain, muscle strength and duration of delirium (expressed in days of ICU or hospital stay), the standardised mean differences or the mean differences with $95 \%$ CIs were calculated, as recommended by the Cochrane Handbook. ${ }^{17}$ Adverse events were narratively summarised because their definition often varies across studies. We used the random-effects models for all analyses.

We calculated $\mathrm{I}^{2}$ as a measure of variation across studies that is due to heterogeneity rather than chance, and interpreted the values as follows: $0 \%-40 \%$, negligible heterogeneity; 30\%-60\%, mild-to-moderate heterogeneity; 50\%-90\%, moderate-to-substantial heterogeneity; $75 \%-100 \%$, considerable heterogeneity. If heterogeneity was identified for an outcome $\left(\mathrm{I}^{2}>50 \%\right)$, we investigated the underlying reasons and conducted the $\chi^{2}$ test, with a $p$ value of $<0.10$ being considered to indicate statistical significance. We investigated reporting bias by checking the WHO ICTRP to detect trials that had been completed but not published at the time of the review.

We planned the following prespecified sensitivity analyses for the primary outcomes: (i) exclusion of studies using imputed statistics and (ii) exclusion of studies with high or unclear risk of bias. We also carried out prespecified subgroup analyses according to the type of rehabilitation involved (neuromuscular stimulation vs other types of rehabilitation), rehabilitation provision in the ICU (received vs did not receive protocolised physical rehabilitation in the ICU), timing of commencement of the intervention (in-hospital or after hospital discharge), intervention duration ( $\leq 8 \mathrm{vs}>8$ weeks), treatment frequency ( $<5 \mathrm{vs} \geq 5$ times/week) and type of control (no intervention vs standard rehabilitation). Statistical significance was also set at $\mathrm{p}<0.05$. We created a summary-of-findings table that included an overall grading of the certainty of evidence for each of the main outcomes, which was evaluated using the Grading of Recommendations, Assessment, Development and Evaluation (GRADE) approach. $^{2021}$

\section{Patient and public involvement}

The patients or public were not involved in this meta-analysis.

\section{RESULTS}

\section{Characteristics of trials on rehabilitation in ICU survivors}

After removing duplicates, we identified 3589 records during the search conducted in December 2017 and updated the electronic searches in January 2019. We identified 10 unique RCTs ${ }^{11-13} 15$ 22-27 that fulfilled all eligibility criteria and were included in the qualitative synthesis (figure 1; details provided in online supplementary file 3 ). The 10 RCTs provided a pooled sample of 1110 critically ill patients with an ICU stay of $>48$ hours during which mechanical ventilation was provided for at least 24 hours. Eight studies were performed in the UK, one in Australia and one in India. The mean or median age in the analysed studies ranged from 40.5 to 68.5 years, while the mean or median Acute Physiology and Chronic Health Evaluation II score ranged from 15.2 to 31. Only one RCT included participants with PICS symptoms or ICU-acquired weakness. ${ }^{11}$ Three RCTs ${ }^{25-27}$ did not have sufficient outcome data for meta-analysis (details provided in online supplementary file 4), leaving a total pooled sample of 1000 patients (506 patients in the intervention groups; 494 controls) represented across seven studies to be included in the quantitative synthesis. Of the 10 trials analysed, 6 evaluated the effect of physical rehabilitation including self-directed exercise and/or supervised exercise following hospital discharge, while $4^{1222-24}$ focused on rehabilitation started during hospitalisation. The duration of intervention ranged from 6 weeks to 3 months, while the frequency of intervention ranged from three times per week to once daily. No study considered intensive intervention $(>30 \mathrm{~min}$ of active rehabilitation daily) or intervention with neuromuscular stimulation. Two studies ${ }^{12}{ }^{23}$ had a follow-up $>6$ months. We did not identify any ongoing studies.

Most studies were at high or unclear risk of bias, as determined using the Cochrane risk-of-bias assessment tool $^{17}$ (details provided in online supplementary file 5). All 10 studies demonstrated adequate random sequence generation and allocation concealment, but participants and personnel were not blinded to the intervention. One study ${ }^{11}$ demonstrated a high risk of detection bias for all outcomes except mortality, and another study ${ }^{27}$ did not report whether or not the outcome assessor was aware of group allocation. Five studies had high risk of incomplete outcome data. Four studies had high risk of selective reporting bias, and two studies had unclear risk of bias because the protocols were not published. High or unclear risk of other bias was noted for all studies because of insufficient information regarding the intervention and control protocols.

\section{Primary outcomes}

QOL was measured in nine trials (see online supplementary file 3), but the short-term and long-term QOL 


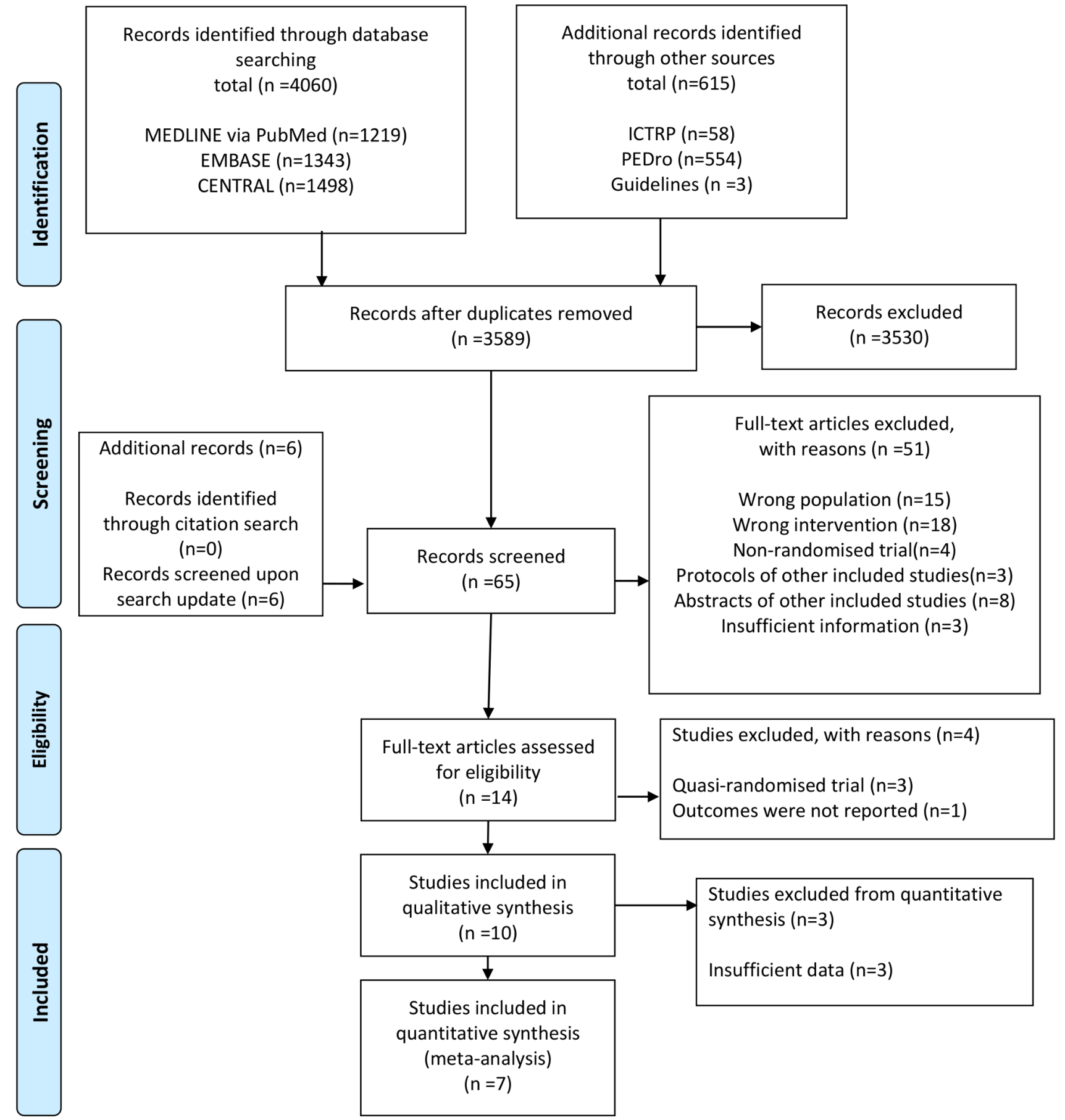

Figure 1 Preferred Reporting Items for Systematic reviews and Meta-Analyses flow diagram.

scores were only available in four trials, ${ }^{122-24}$ whereas the other five trials measured these outcomes at a different time or had insufficient outcome data for meta-analysis. ADL function was measured in one trial, ${ }^{11}$ but the shortterm and long-term data were not available. Short-term mortality was reported in two trials, ${ }^{11}{ }^{13}$ while long-term mortality was reported in five trials. ${ }^{12} 15$ 22-24

The standard mean deviation between intervention and control regarding the physical and mental component summary scores measured using QOL questionnaires (Short Form 36 or Short Form 12) were 0.06 (95\% CI -0.12 to 0.24$)$ and -0.04 (95\% CI -0.20 to 0.11 ), respectively (figure 2A,B respectively). Rehabilitation did not significantly decrease short-term mortality (RR $0.71 ; 95 \%$ CI 0.05 to $9.80, \mathrm{I}^{2}=33 \%$; $=93$ ) (figure $2 \mathrm{C}$ ) or long-term mortality (RR 1.05 ; $95 \%$ CI 0.66 to $1.66, \mathrm{I}^{2}=0 \%$; $\mathrm{n}=907$ ) (figure 2D). The certainty of evidence for QOL and long-term mortality was moderate, while that for short-term mortality was low (table 1). The lack of benefit of enhanced physical rehabilitation after ICU discharge was confirmed on additional analysis of QOL scores and mortality at 12 months postintervention (see details provided in online supplementary file 6 ). 
A Quality of life: physical component summary

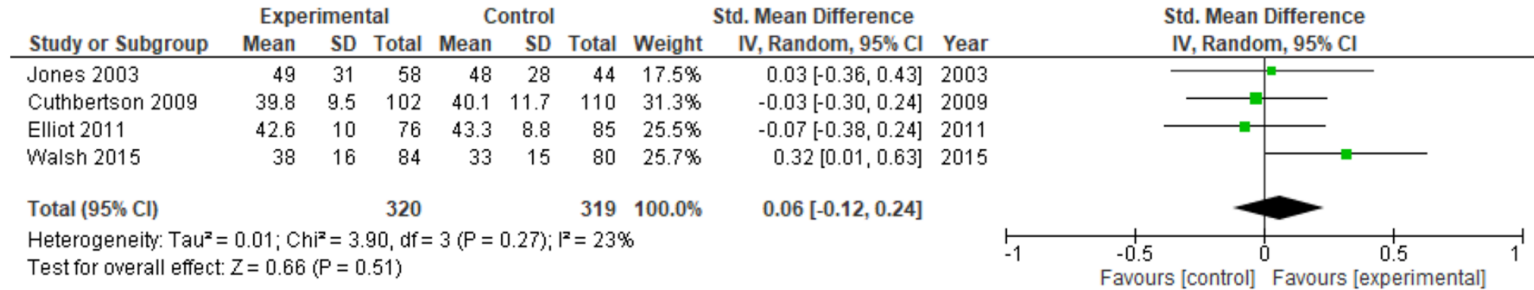

B Quality of life: mental component summary

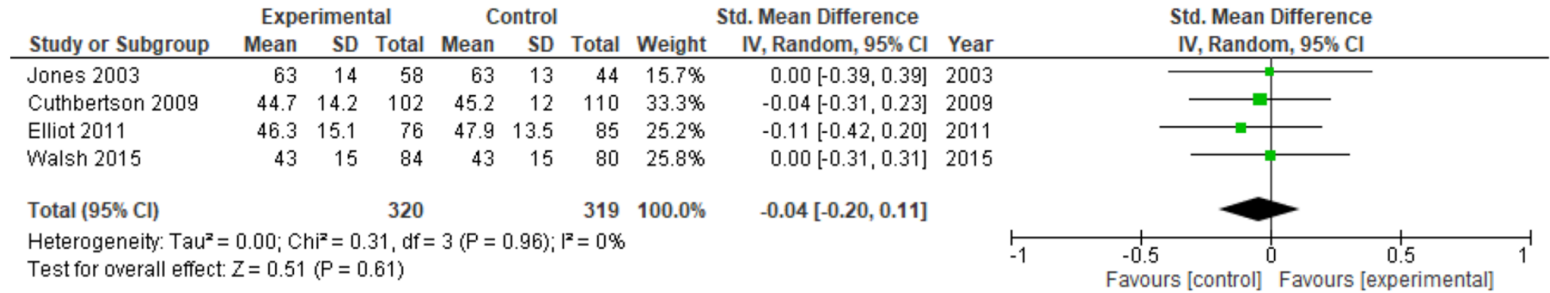

C Short term mortality

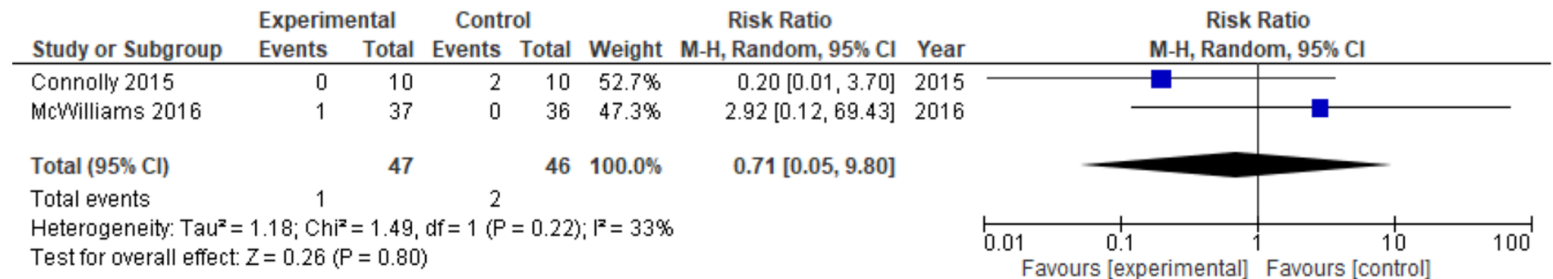

D Long term mortality

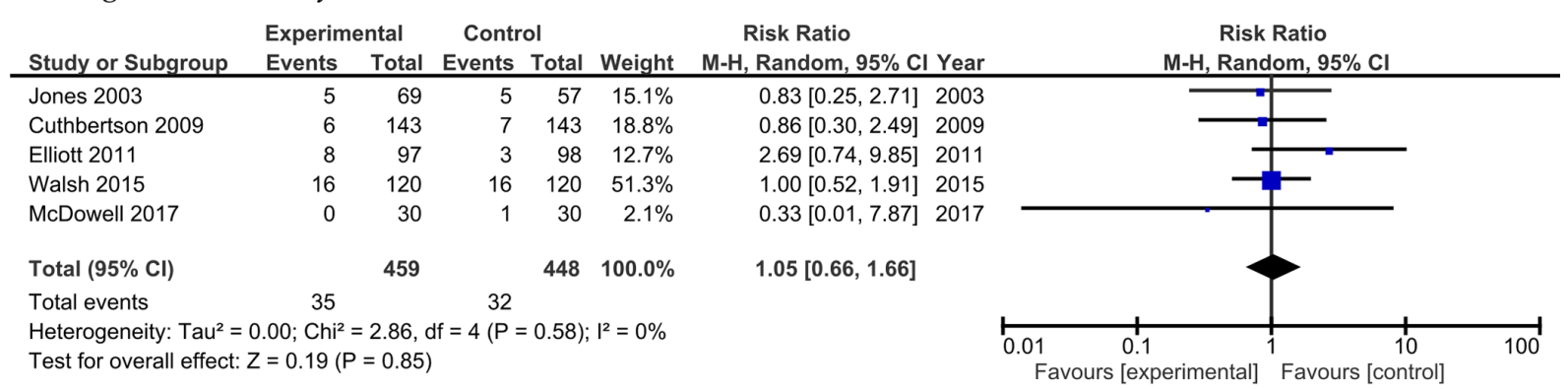

We converted median (inter quartile range) of QOL score in Walsh's study to mean (standard deviation).

Figure 2 Forest plot for quality of life and mortality.

We could not carry out all prespecified sensitivity analyses because there was no study using imputed statistics, and we judged that the risk of bias of all included studies was similar in terms of random sequence generation, allocation concealment, incomplete outcome data and other bias. The prespecified subgroup analyses for the primary outcomes revealed no significant differences among subgroups (see details provided in online supplementary file 7).

\section{Secondary outcomes}

Functional exercise capacity was measured in two trials, ${ }^{11} 24$ pain was measured in one trial ${ }^{12}$ and muscle strength was measured in one trial, ${ }^{11}$ but short-term and long-term data were not available. No trials evaluated return-to-work rate or incidence of delirium.
Adverse events were measured in three trials. ${ }^{11} 1315$ Two studies ${ }^{11} 13$ reported no adverse events. One study ${ }^{15}$ reported 18 events in the intervention group and 5 events in the control group. Among the 18 adverse events reported in the intervention group, 12 were mild or moderate (musculoskeletal pain higher than expected or muscle soreness potentially indicating injury, 3 cases; any pain higher than expected, 1 case; cardiac symptoms or chest pain, 1 case; any other event considered by the researcher to be of concern, 7 cases; 6 of 12 events were considered to be related or possibly related to study participation), while 6 were serious (hospitalisation or prolonged hospitalisation, with one event related/ possibly related to study participation). In the control group, there was one adverse event (musculoskeletal 


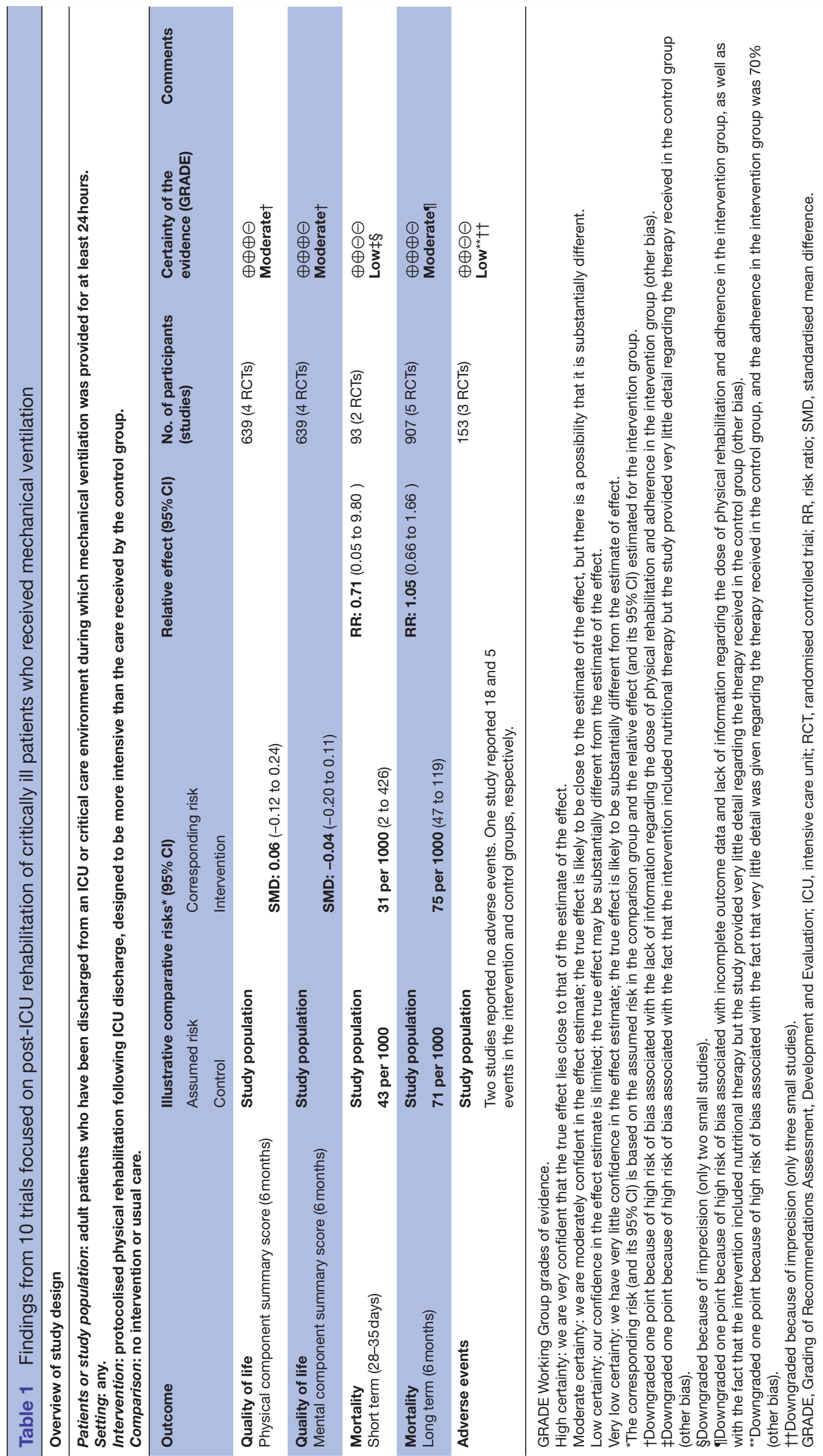


pain higher than expected, muscle soreness potentially indicating injury, related/possibly related to study participation) and four serious adverse events (hospitalisation or prolonged hospitalisation, with one event related/ possibly related to study participation). The certainty of evidence for adverse events was low (table 1).

\section{DISCUSSION}

The results of this up-to-date review covering 10 RCTs and 1110 patients suggest that enhanced rehabilitation following ICU discharge might not improve QOL or reduce mortality at 6 or 12 months postintervention among patients who received mechanical ventilation in the ICU. We could not confirm the effect of enhanced physical rehabilitation even though all included studies exhibited performance bias potentially increasing the observed effect of the intervention. Furthermore, despite the large sample size in the meta-analysis for QOL and long-term mortality, limited data for these outcomes were available, and the certainty of evidence was only low or moderate.

Furthermore, subgroup meta-analyses revealed no differences among subgroups defined according to the nature or timing of the intervention. The previous review by Connolly et al ${ }^{10}$ did not conduct meta-analysis due to the limited number of included studies. A recent systematic review of ICU rehabilitation ${ }^{28}{ }^{29}$ also reported no significant difference in QOL between the intervention and control groups. Thus, neither enhanced rehabilitation in the ICU nor rehabilitation following ICU discharge appear to be superior to standard care in terms of QOL outcomes. In addition, we found no benefit in terms of short-term or long-term mortality regardless of timing of commencement, which is consistent with previous findings that ICU rehabilitation did not decrease mortality at ICU discharge, at hospital discharge or at 6 months after discharge..$^{28}{ }^{30}$ On the other hand, rehabilitation may be detrimental in acute conditions. Specifically, intensive physical rehabilitation started within 48 hours of admission for exacerbations of chronic respiratory disease increased mortality at 12 months, ${ }^{31}$ and higher-dose physical rehabilitation very early after stroke decreased favourable outcomes at 3 months. ${ }^{32}$ Thus, implementation of an intensive rehabilitation programme might not be indicated in all patients who received mechanical ventilation in the ICU.

Subgroup analysis in a previous systematic review ${ }^{28}$ indicated that, compared with low-dose rehabilitation, highdose active rehabilitation for $>30$ min daily was associated with significantly higher QOL. Dose-response analysis of early physical rehabilitation ${ }^{33}$ in patients with stroke enrolled in A Very Early Rehabilitation Trial ${ }^{32}$ determined that intervention in such acute cases improved the odds of a favourable outcome with each episode of activity per day. Our present review did not include studies comparing high-dose rehabilitation and usual care, and thus the QOL effect of high-dose rehabilitation remains unclear.
Additionally, we could not perform subgroup analysis for PICS symptoms with effect on $\mathrm{QOL}^{3-5}$ or for sepsis, which is a risk factor for PICS. ${ }^{34} 35$ It remains unclear which population of critically ill patients may truly benefit from intensive physical rehabilitation.

The studies included in our review did not cover all important outcomes included in the core outcome set of rehabilitation after critical illness, ${ }^{7}$ including ADL function, functional exercise capacity, pain, return-to-work rate, muscle strength or delirium incidence. Nonetheless, our findings regarding QOL and mortality suggest that, even if future studies report improvement in these other aspects, the amount of improvement would likely be too small to affect QOL.

The present review has several strengths. First, we employed rigorous methodology that followed a written protocol developed a priori according to the PRISMA statement, including a comprehensive search for evidence. Second, we performed duplicate assessment of eligibility, risk of bias and data abstraction. Third, we used the GRADE approach for assessing the certainty of evidence. In addition, we only included RCTs, most of which were multicentre studies. We could thus conduct an intention-to-treat analysis to understand the effect of intensive physical rehabilitation or standard care, which gives a pragmatic estimate of the benefit of a change in treatment policy. Fourth, the cohorts of ICU survivors are heterogeneous in terms of demographics and pathologies. To confirm the effect of enhanced physical rehabilitation for a particular group, we selected studies including only participants with an ICU stay of $>48$ hours during which mechanical ventilation was provided for at least 24 hours.

This systematic review has several potential limitations. First, few studies ${ }^{12} 23$ had a follow-up $>6$ months, and thus we could not consider longer follow-up data for primary analysis. The meta-analysis should be updated as the outcomes of further studies with follow-up beyond 6 months become available. Second, none of the studies included in our meta-analysis reported mortality outcomes as time-to-event data, which is the preferred approach for reporting mortality data. Future studies should report time-to-event data for mortality. Third, we could not take into account the medical resources and costs associated with each intervention. However, since studies included in this review compare rehabilitation intervention against standard care or no intervention, it is obvious that intensive physical rehabilitation would be associated with increased medical resources and costs. Fourth, the outcome measures might be not sufficiently sophisticated. For example, the RECOVER (Evaluation of a Rehabilitation Complex Intervention for Patients Following Intensive Care Discharge) trial $^{12}$ did not demonstrate an improvement in the primary quantitative outcome, but showed evidence of benefit of the intervention in a parallel qualitative evaluation. ${ }^{36}$ Fifth, we could not consider the psychological aspects that are likely to affect the outcomes of rehabilitation. While our findings 
indicate a lack of benefit of enhanced post-ICU rehabilitation in the evaluated population, highly self-motivated individuals might have derived benefit from such therapies. Further studies should collect data on motivation and engagement, which are crucial in maximising the benefits of rehabilitation. ${ }^{37}$ Lastly, the patient characteristics, follow-up timing and types of outcomes reported might exhibit substantial heterogeneity across trials and within each individual trial, an aspect we did not examine in the present analysis. However, on reviewing the best available evidence based on a standardised approach, we confirmed that the direction of the effect and the effect size of enhanced post-ICU physical rehabilitation were similar in pooled studies, as reflected in the forest plots (see details in online supplementary file 7 ).

Taken together, the findings of the present meta-analysis indicate that enhanced physical rehabilitation following ICU discharge may make little or no difference to QOL or mortality among patients who received mechanical ventilation in the ICU. Given the wide CIs, further studies are needed to determine the efficacy of enhanced rehabilitation in selected populations of ICU survivors.

\section{Author affiliations}

${ }^{1}$ Hiroshima University Hospital, Hiroshima, Japan

${ }^{2}$ Department of Rehabilitation, Steel Memorial Yawata Hospital, Fukuoka, Japan

${ }^{3}$ Healthcare Epidemiology, School of Public Health in the Graduate School of

Medicine, Kyoto University, Kyoto, Japan

${ }^{4}$ Nephrology and Dialysis, Kyoritsu Hospital, Kawanishi, Japan

${ }^{5}$ Department of Psychiatry, Seichiryo Hospital, Aichi, Japan

${ }^{6}$ Department of Psychiatry, Nagoya University, Graduate School of Medicine, Nagoya, Japan

${ }^{7}$ Hospital Care Research Unit, Hyogo Prefectural Amagasaki General Medical Center, Amagasaki, Japan

${ }^{8}$ Hospital Care Research Unit, Hyogo Prefectural Amagasaki Hospital, Amagasaki, Japan

${ }^{9}$ Hyogo Prefectural Amagasaki Hospital, Amagasaki, Japan

Acknowledgements The authors would like to thank Dr Avelino C. Verceles (Division of Pulmonary, Critical Care and Sleep Medicine, University of Maryland School of Medicine), Ms Bernie Bissett (Canberra Hospital), Dr Bronwen Connolly (St Thomas' Hospital), Dr Yen-Huey Chen (Department of Respiratory Therapy, Chang Gung University), Dr Christina Jones (Whiston Hospital), Mr Danny Martin (Department of Physical Therapy, University of Florida), Dr Jennifer Paratz (Burns, Trauma \& Critical Care Research Centre, School of Medicine, University of Queensland), Dr Kensuke Nakamura (Hitachi General Hospital), Kirstine Sibilitz (Department of Cardiology, Hvidovre University Hospital), Ms Ling Ling Chiang (School of Respiratory Therapy, Taipei Medical University), Ms Lisa Salisbury (Dietetics, Nutrition \& Biological Sciences, Physiotherapy, Podiatry \& Radiography Division, Queen Margaret University), Dr Michele Vitacca (Istituti Clinici Scientifici Maugeri), Dr Richard D Griffiths (Institute of Ageing and Chronic Disease, University of Liverpool), Mr Rik Gosselink (Faculty of Kinesiology and Rehabilitation Science, University of Leuven), Ms Seher Özyürek (School of Physical Therapy and Rehabilitation, Dokuz Eylul University), Ms Sunita Mathur (Department of Physical Therapy, University of Toronto) and Dr Timothy S. Walsh (Anaesthesia, Critical Care and Pain Medicine, University of Edinburgh) for providing us with additional information regarding their studies. The authors would like to thank Editage (http:// www.editage.jp) for English language editing.

Contributors ST and KY designed the study, were involved in the systematic review process, analysed and interpreted the data and drafted the manuscript. MB, $\mathrm{HT}$, YK and YT participated in the systematic review process, critically reviewed the initial manuscript and approved the final manuscript as submitted. All authors read and approved the final manuscript.

Funding This work was supported by JSPS KAKENHI Grant Number JP18K17719.
Competing interests None declared.

Patient consent for publication Obtained.

Provenance and peer review Not commissioned; externally peer reviewed.

Data sharing statement All data associated with this manuscript are included in the main text and supplementary materials.

Open access This is an open access article distributed in accordance with the Creative Commons Attribution Non Commercial (CC BY-NC 4.0) license, which permits others to distribute, remix, adapt, build upon this work non-commercially, and license their derivative works on different terms, provided the original work is properly cited, appropriate credit is given, any changes made indicated, and the use is non-commercial. See: http://creativecommons.org/licenses/by-nc/4.0/.

\section{REFERENCES}

1. World Health Organization. International classification of functioning, disability and health (ICF). 2001. http://www.who.int/classifications/ icf/en/ [Accessed 24 May 2018]

2. Hodgson CL, Udy AA, Bailey M, et al. The impact of disability in survivors of critical illness. Intensive Care Med 2017;43:992-1001.

3. Fan E, Dowdy DW, Colantuoni E, et al. Physical complications in acute lung injury survivors: a two-year longitudinal prospective study. Crit Care Med 2014;42:849-59.

4. Hermans G, Van Mechelen H, Clerckx B, et al. Acute outcomes and 1-year mortality of intensive care unit-acquired weakness. A cohort study and propensity-matched analysis. Am J Respir Crit Care Med 2014;190:410-20.

5. Naidech AM, Beaumont JL, Rosenberg NF, et al. Intracerebral hemorrhage and delirium symptoms. Length of stay, function, and quality of life in a 114-patient cohort. Am J Respir Crit Care Med 2013;188:1331-7.

6. National Institute for Health and Care Excellence. Rehabilitation after critical illness. 2008. https://www.nice.org.uk/guidance/cg83 [Accessed 24 May 2018].

7. Major ME, Kwakman R, Kho ME, et al. Surviving critical illness: what is next? An expert consensus statement on physical rehabilitation after hospital discharge. Crit Care 2016;20:354.

8. Prescott HC, Angus DC. Enhancing recovery from sepsis: a review. JAMA 2018;319:62-75.

9. Chao PW, Shih CJ, Lee YJ, et al. Association of postdischarge rehabilitation with mortality in intensive care unit survivors of sepsis. Am J Respir Crit Care Med 2014;190:1003-11.

10. Connolly B, Salisbury L, O'Neill B, et al. Exercise rehabilitation following intensive care unit discharge for recovery from critical illness. Cochrane Database Syst Rev 2015:CD008632.

11. Connolly B, Thompson A, Douiri A, et al. Exercise-based rehabilitation after hospital discharge for survivors of critical illness with intensive care unit-acquired weakness: a pilot feasibility trial. $J$ Crit Care 2015;30:589-98

12. Walsh TS, Salisbury LG, Merriweather JL, et al. Increased hospitalbased physical rehabilitation and information provision after intensive care unit discharge: the RECOVER randomized clinical trial. JAMA Intern Med 2015;175:901-10.

13. McWilliams DJ, Benington S, Atkinson D. Outpatient-based physical rehabilitation for survivors of prolonged critical illness: a randomized controlled trial. Physiother Theory Pract 2016;32:179-90.

14. Patsaki I, Gerovasili V, Sidiras G, et al. Effect of neuromuscular stimulation and individualized rehabilitation on muscle strength in intensive care unit survivors: a randomized trial. J Crit Care 2017;40:76-82.

15. McDowell K, O'Neill B, Blackwood B, et al. Effectiveness of an exercise programme on physical function in patients discharged from hospital following critical illness: a randomised controlled trial (the REVIVE trial). Thorax 2017;72:594.1-5.

16. Taito S, Yamauchi K, Tsujimoto Y, et al. Systematic review and meta-analysis of physical rehabilitation following intensive care unit discharge. 2018. https://www.crd.york.ac.uk/PROSPERO/display_ record.php?RecordID=80532 [Accessed 24 May 2018].

17. Higgins JPT, Green S, eds. Cochrane handbook for systematic reviews of interventions, version 5.1.0. 2011. http://handbook-5-1. cochrane.org/ [Accessed 24 May 2018].

18. Liberati A, Altman DG, Tetzlaff J, et al. The PRISMA statement for reporting systematic reviews and meta-analyses of studies that evaluate health care interventions: explanation and elaboration. PLOS Med 2009;6:e1000100. 
19. Moher D, Liberati A, Tetzlaff J, et al. Preferred reporting items for systematic reviews and meta-analyses: the PRISMA statement. PLoS Med 2009;6:e1000097.

20. Guyatt GH, Oxman AD, Vist GE, et al. GRADE: an emerging consensus on rating quality of evidence and strength of recommendations. BMJ 2008;336:924-6.

21. Guyatt G, Oxman AD, Akl EA, et al. GRADE guidelines: 1. Introduction-GRADE evidence profiles and summary of findings tables. J Clin Epidemiol 2011;64:383-94.

22. Jones C, Skirrow P, Griffiths RD, et al. Rehabilitation after critical illness: a randomized, controlled trial. Crit Care Med 2003;31:2456-61.

23. Cuthbertson BH, Rattray J, Campbell MK, et al. The PRaCTICaL study of nurse led, intensive care follow-up programmes for improving long term outcomes from critical illness: a pragmatic randomised controlled trial. BMJ 2009;339:b3723.

24. Elliott D, McKinley S, Alison J, et al. Health-related quality of life and physical recovery after a critical illness: a multi-centre randomised controlled trial of a home-based physical rehabilitation program. Crit Care 2011;15:R142.

25. Salisbury LG, Merriweather JL, Walsh TS. The development and feasibility of a ward-based physiotherapy and nutritional rehabilitation package for people experiencing critical illness. Clin Rehabil 2010;24:489-500.

26. Batterham AM, Bonner S, Wright J, et al. Effect of supervised aerobic exercise rehabilitation on physical fitness and quality-of-life in survivors of critical illness: an exploratory minimized controlled trial (PIX study). Br J Anaesth 2014;113:130-7.

27. Shelly AG, Prabhu NS, Jirange P, et al. Quality of life improves with individualized home-based exercises in critical care survivors. Indian J Crit Care Med 2017:21:89-93

28. Tipping CJ, Harrold M, Holland A, et al. The effects of active mobilisation and rehabilitation in ICU on mortality and function: a systematic review. Intensive Care Med 2017;43:171-83.
29. Fuke R, Hifumi T, Kondo $\mathrm{Y}$, et al. Early rehabilitation to prevent postintensive care syndrome in patients with critical illness: a systematic review and meta-analysis. BMJ Open 2018;8:e019998.

30. Girard TD, Alhazzani W, Kress JP, et al. An official American Thoracic Society/American College of Chest Physicians clinical practice guideline: liberation from mechanical ventilation in critically ill adults. Rehabilitation protocols, ventilator liberation protocols, and cuff leak tests. Am J Respir Crit Care Med 2017:195:120-33.

31. Greening NJ, Williams JE, Hussain SF, et al. An early rehabilitation intervention to enhance recovery during hospital admission for an exacerbation of chronic respiratory disease: randomised controlled trial. BMJ 2014;349:g4315.

32. AVERT Trial Collaboration Group. Efficacy and safety of very early mobilisation within $24 \mathrm{~h}$ of stroke onset (AVERT): a randomised controlled trial. Lancet 2015;386:46-55.

33. Bernhardt J, Churilov L, Ellery F, et al. Prespecified dose-response analysis for A Very Early Rehabilitation Trial (AVERT). Neurology 2016;86:2138-45.

34. Stevens RD, Dowdy DW, Michaels RK, et al. Neuromuscular dysfunction acquired in critical illness: a systematic review. Intensive Care Med 2007;33:1876-91.

35. Iwashyna TJ, Ely EW, Smith DM, et al. Long-term cognitive impairment and functional disability among survivors of severe sepsis. JAMA 2010;304:1787-94.

36. Ramsay P, Huby G, Merriweather J, et al. Patient and carer experience of hospital-based rehabilitation from intensive care to hospital discharge: mixed methods process evaluation of the RECOVER randomised clinical trial. BMJ Open 2016;6:e012041.

37. Corner EJ, Murray EJ, Brett SJ. Qualitative, grounded theory exploration of patients' experience of early mobilisation, rehabilitation and recovery after critical illness. BMJ Open 2019;9:e026348. 